International Journal of Engineering \& Technology, $7(3.16)(2018) 81-84$
International Journal of Engineering \& Technology
SPC
Website: www.sciencepubco.com/index.php/IJET
Research paper

\title{
Energy Consumption Behaviors of 802.11 and S-MAC: Implementation on NS2.35
}

\author{
Meena Malik ${ }^{1 *}$, Mukesh Sharma ${ }^{2}$ \\ ${ }^{1}$ Department of Computer Science and Engineering, Maharishi Dayanand University \\ Rohtak, Haryana, 124001, India \\ ${ }^{2}$ Department of Computer Science \& Engineering The Technological Institute of Textile \& Sciences \\ Bhiwani, Haryana 127021 India \\ *Corresponding author E-mail:meenamlk@gmail.com
}

\begin{abstract}
The Sensor technology has made encouraging trends in the field of wireless Networks by its innovative methods and adaptability. The fundamental issue for wireless sensor networks (WSN) is to minimize energy consumption at each node due to restricted energy source. The sensor nodes generally get random deployment and need cooperation to accomplish specific operation in the network like monitoring or tracking any target in the environment. Due to limited power source nodes need careful use of energy resources. This work targets on simulating the power consumption behavior and analyzing the performance of 802.11 and S-MAC protocol for medium access control layer in wireless networks. S-MAC improves energy consumption by allocating bandwidth in efficient manner and avoiding causes of energy waste. After simulation, it was found that S-MAC is Power-Efficient over 802.11 without losing on the performance using NS-2.35. The paper mainly emphasize on representing the plot for energy matrices along with throughput, delay and packet delivery ratio.
\end{abstract}

Keywords: Energy Consumption, S-MAC, Wireless Sensor Network, 802.11 MAC.

\section{Introduction}

This Wireless Sensor Network (WSN) is a network of large number of sensor devices monitor environmental conditions such as humidity, pressure, temperature, vibration noise, motion, or pollution. The major problematic issue for wireless networks is energy consumption because every sensing device operates by an inadequate capability battery source[1]. The deployment of sensor nodes is generally random scatter in the target area. The MAC layer is responsible to establish a communication and transferring data in order to achieve fair channel allocation and reduction in energy consumption. Energy of sensor node is a very tough issue due to hardware constraints. We know the fact, more energy is spent by the node active in communication rather the other nodes. To enhance lifetime of the network, it is surely essential to employ energy aware and efficient protocols for communication in WSNs[2]. The Wireless property of the network results in the channel through which communication should occur, be a shared media and arise need of significantly efficient protocol. The efficient communications require significant amount of energy and further will ultimately result in lowers the total energy in the network. All these reasons lead to choose and design a power aware protocol at MAC layer in WSNs so as to increase network lifetime and throughput[3]. From last decade, researchers have introduced many MAC protocols in order to control sources of energy wastes like message passing, overhearing avoidance and idle listening [1]. This paper describes the simulation of energy consumption behavior in the network using 802.11 and S-MAC and thereby plotting results. In Section 2 a brief background of both the considered protocols is presented. After that Section 3 explains about network simulator and tabulates all the parameters and assumptions used in simulation following by Section 4 that plots results and analyze both protocols on the behalf of all parameters followed by discussion of results and finally conclusion is done.

\section{Background of 802.11 and S-MAC}

The protocols at MAC layer are categorized into main three types: Based on CSMA, TDMA and hybrid approach. In Carrier Sense Multiple Access (CSMA) protocols, a node first attends channel assessment process to check whether the channel is free or busy due to transmissions with other node. When a node get a new packet for transmission on the channel, it is sensed and if found idle the packet is transmitted.

In case a collision occurs due to multiple nodes transmitting, rescheduling for retransmission of the collided packet is done after a random span. In contrast[4], CSMA results in lesser delay and capable of potential throughput at lower traffic loads, that is required in WSNs. In TDMA, (Schedule-Based Protocols ) numerous users are permissible to segment the identical frequency channel and the signal is separated into different time-slots. Due to different time slot of individually nodes TDMA offers collision free medium access. All nodes lead to turning ON the radio during its allotted slot and results into low duty cycle operation. However, it includes clock drift problems and decreased throughput at low traffic loads due to idle slots. 
Standard 802.11[5][6], is a contention based MAC protocol for executing wireless local area network (WLAN). The collisions of packets is controlled by carrier sensing and randomized back-offs algorithm. It is very simple to implement, flexibility in architecture and a cost effective method for channel allocation. IEEE 802.11 with distributed coordination function (DCF) mainly apply Fragmentation mechanism virtual carrier sense, binary exponential back-off in the networks for efficient functioning[1]. IEEE 802.11 was fundamentally designed for one hop network [8]. It works efficiently in powered networks due to its basic characteristics like high speed bit rates and fairness but due to long idle listening, and imprecise sleep period strategy it is not suitable for WSN.

S-MAC[7] is a medium access control protocol that focuses on energy efficient for wireless sensor networks and allows peer to peer communication between the nodes present in the network. It offers the notion of distributing the whole frame into phase of listen and sleep periods. During a listen phase, the communication follows through all the concerned nodes and afterward next phase is sleep that puts radio fully turned off and wait for a certain time to get alert. The next cycle repeats similar procedure and again node appears to check whether any communication is there from other node. The needed communication is completed in short duration of listen phase exchanging SYNC, Request-To-Send, and Clear-To-Send packets. The Nodes that are involved in message exchange continue awake and every node grow permitted to choose the listen-sleep schedules by their own. After deciding corresponding schedules nodes share their information about schedules to neighbors in order to achieve efficient communication among all nodes. Now Nodes may schedule broadcasts while their neighboring nodes be vested in the listen phase. The control overhead may be minimized by allowing the neighboring nodes to implement identical schedules. The listen and sleep period duration is decided on behalf of Radio bandwidth and contention window size. In S-MAC[8][9], synchronisation among nodes is not strong and results into the protocol incapability to effectively put up topology variations. The major drawback is degradation of fairness, throughput and latency performance and due to which it is not appropriate for the applications containing short idle periods. The latency is increased due to sleep periods along with increasing hops in the network. The duty cycle of S-MAC protocol is fixed due to which it fails to adjust in traffic variation. The collision avoidance mechanism in S-MAC is similar to that in the IEEE 802.11 DCF. way to comply with the paper formatting requirements is to use this document as a template and simply type your text into it.

\section{Methodology Used and Simulation Scenario}

Network Simulator-2.35 has been used to accomplish experimentation and simulations under operating system - VM Ubuntu 14.04 LTS, RAM used-4.0 GB, OS Type - 64 bit and NS package- ns-allinone-2.35. For simulation, a TCL script has been written for our considered scenario and accordingly topology has been decided. Table 1 represents all the parameters and corresponding values used in the TCL script. After running the script file a trace format get generated in the form of .tr file and by using AWK program( an interpreted programing script) the information is extracted and value of all parameters is plotted. A network comprising of 30 nodes deployed in random fashion has been simulated. Node 17 is taken to be the TCP CBR traffic source node and node 25 as the sink node. Traffic Pattern: Source keeps sending the 512 byte data packets to sink taking pause time of 2 seconds meanwhile. All nodes in the network equipped with a limited energy of 10 Joules. Thus initially network contains $30 * 10=300$ joules total energy. For routing decision AODV routing protocol and for propagation two ray ground propagation models is employed here. As we need to compute residual energy therefore Energy and Radio Model are set ON. The Duty Cycle is set to default value that is $10 \%$. Snapshot 1 and snapshot 2 reflects simulation environment for 802.11 and S-MAC respectively and Table 1 denotes all parameters and values used in experimentation and simulation.

Table 1 enlists all the parameters and their values used in experimentation and simulation

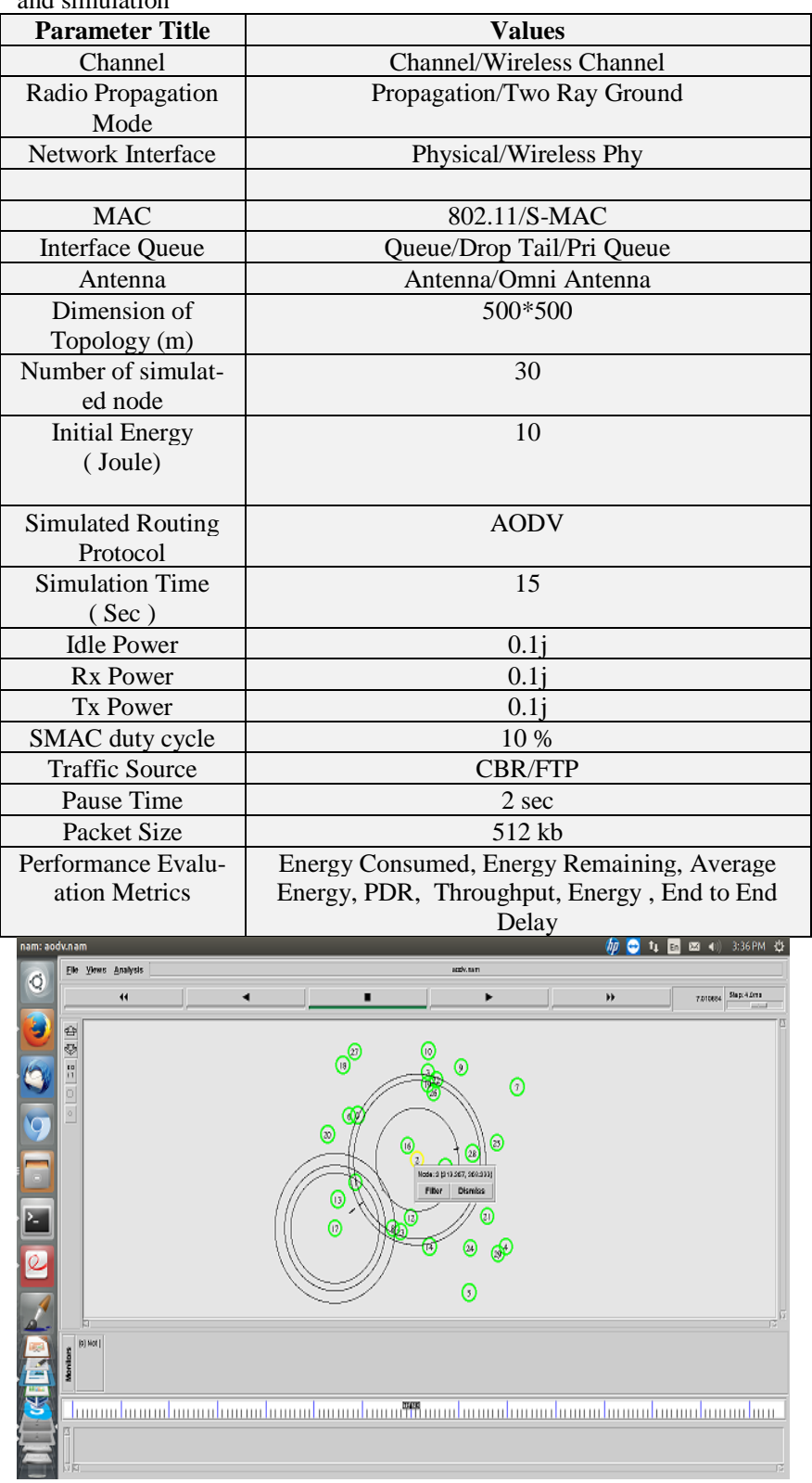

Snapshot1: Topology and Simulation Scenario for 802.11

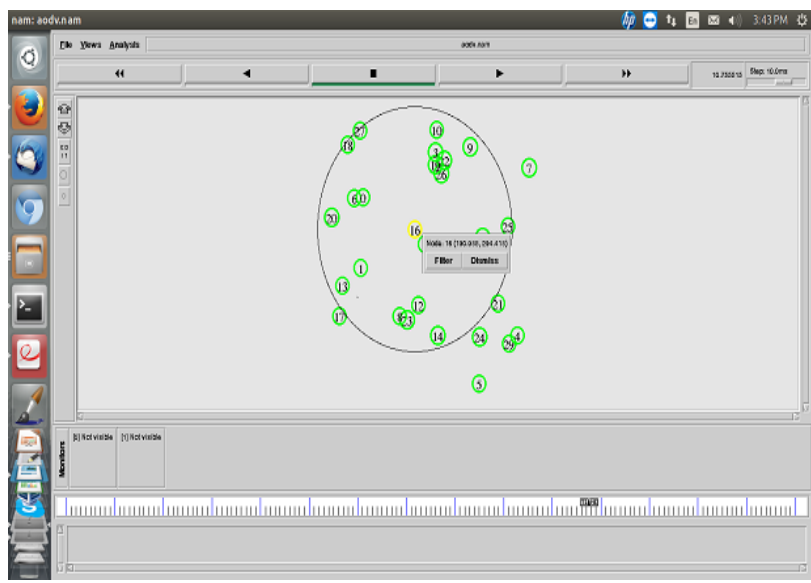

Snapshot 2: Topology and Simulation Scenario for S-MAC 


\section{Results and Analysis}

In 802.11 MAC protocol, nodes are always listening the channel even when the current node does not perform any activity and remains in listen state. But S-MAC[10] follows switching between listen period and sleep period. It means, when the node does not perform any activity, the node switch to sleep period and saves energy by avoiding idle listening.

Energy Consumption : It express the relationship among energy spent to the total of data packets delivered by each node in the network. Figure, we can easily state that 802.11 consumes more energy because radio is always in active state so lifetime of network is short comparatively. S-MAC achieves energy efficiency by switching the radio in SLEEP/ACTIVE state periodically so resulting into energy savings thereby providing longer network life. The Remaining energy in the network is higher and average energy consumption is lower in S-MAC. The implementation results exhibits that the 802.11 MAC protocol consumes more energy than S-MAC.

End-to-End Delay: End to End delay is measure of the ratio between a sum of individual data packet delay to the total number of data packets delivered. Figure illustrates high delay for 802.11 and comparatively very low in S-MAC.

Packet Delivery Ratio(PDR): PDR represents the extent of overall successful delivered data packets from all the sent data packets. PDR for S-MAC is much higher than 802.11.

Throughput: It express the total count of data packets transported to sink node during the decided simulation time. 802.11 outperforms S-MAC for throughput by transporting more data packets to the receiver node. So we can state that S-MAC manages energy efficiency by conceding throughput.

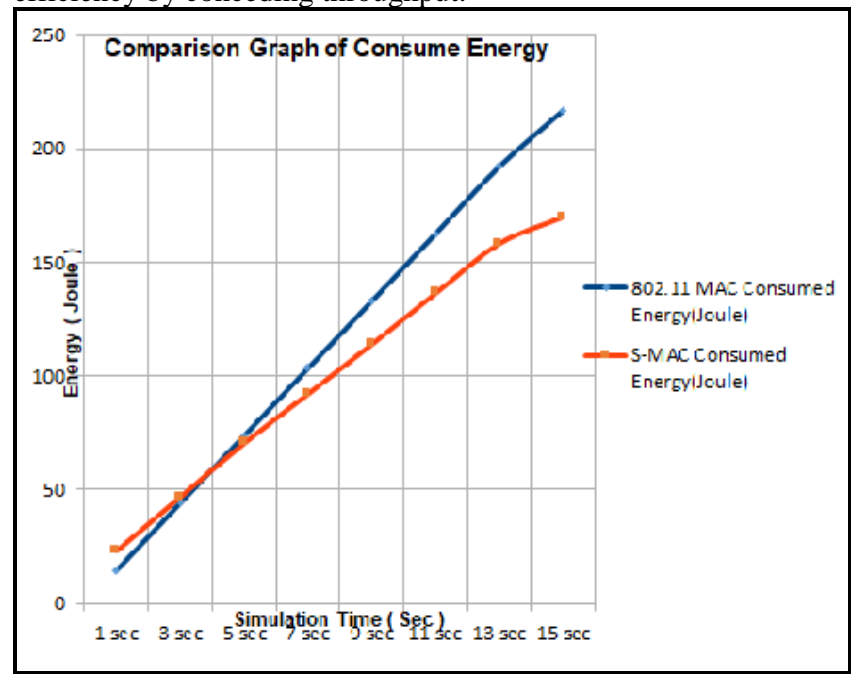

Figure 1: Plots Consumed Energy For 802.11 and S-MAC

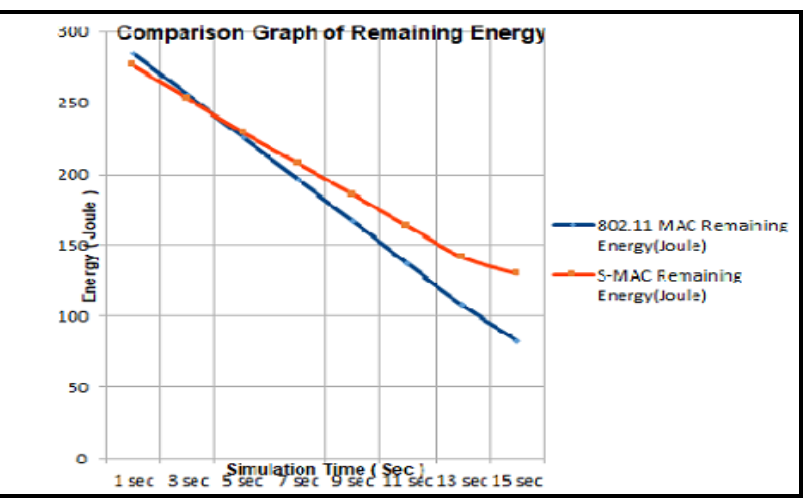

Figure 2: Plots Remaining Energy for 802.11 and S-MAC

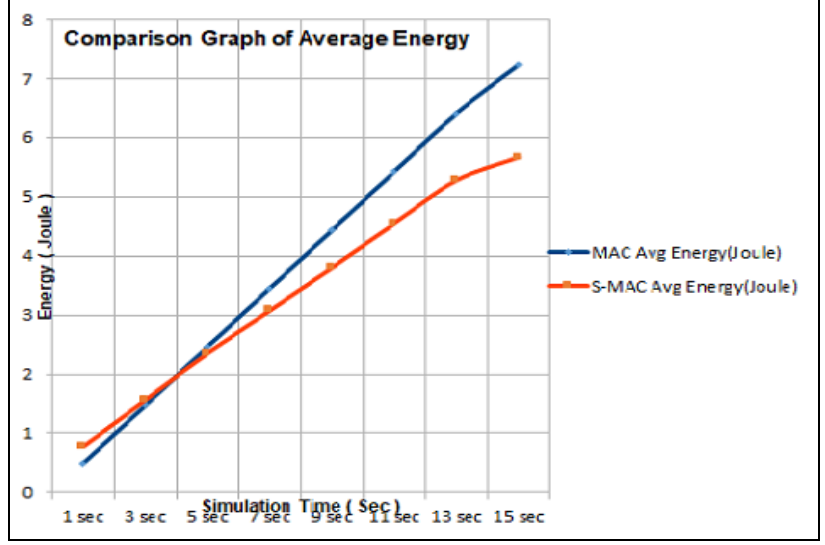

Figure 3: Plots Average energy For 802.11 and S-MAC

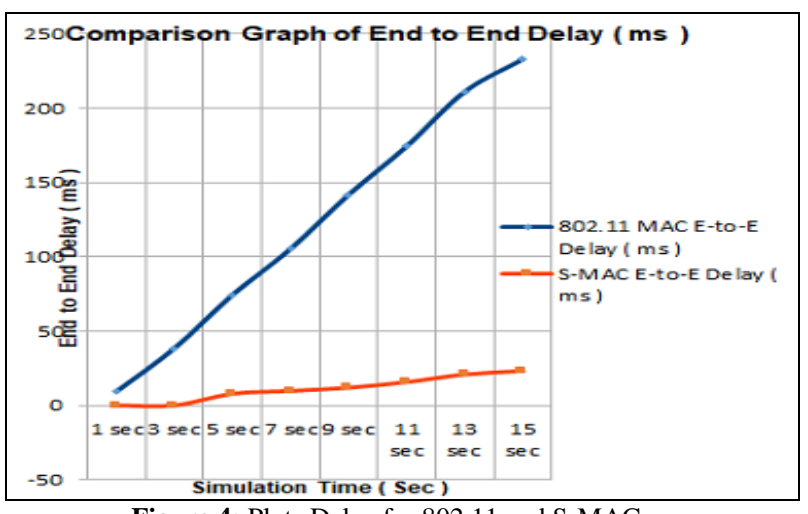

Figure 4: Plots Delay for 802.11 and S-MAC

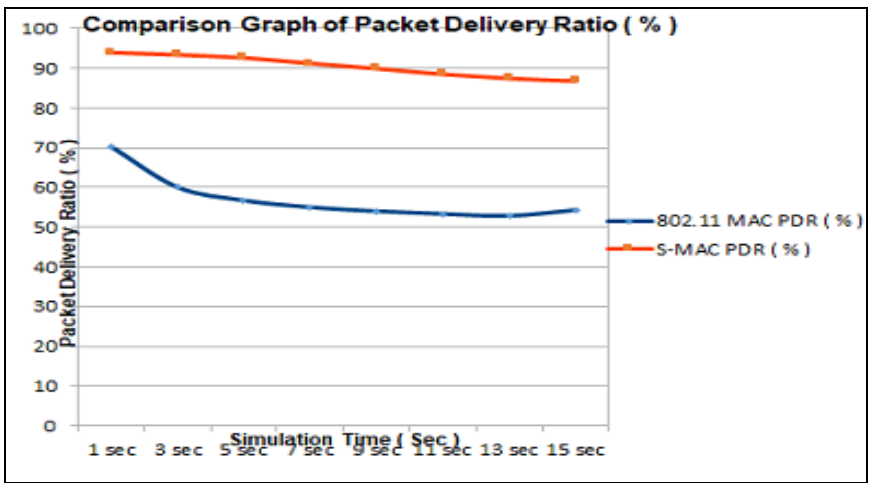

Figure 5: Plots PDR performance for 802.11 and S-MAC

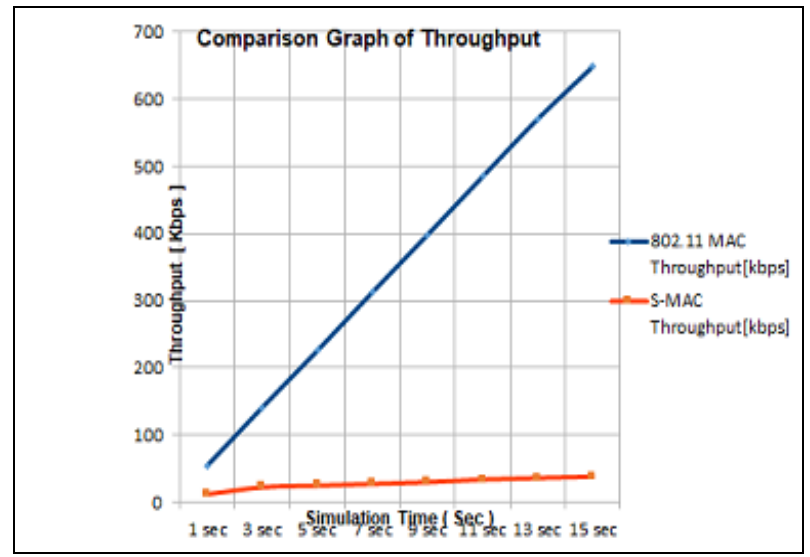

Figure 6: Plots Throughtput performance for 802.11 and S-MAC

Table 2: Illustrates various Simulation Parameters along with evaluation

\begin{tabular}{|c|c|c|c|c|}
\hline Parameter & MAC(802.11) & S-MAC & 802.11 & S-MAC \\
\hline Total Energy (Joule) & 300 & 300 & SAME & SAME \\
\hline Avg Energy(Joule) & 6.99077 & 5.78673 & LOW & LOWER \\
\hline $\begin{array}{c}\text { Consumed Ener- } \\
\text { gy(Joule) }\end{array}$ & 209.723 & 173.602 & HIGH & LOW \\
\hline $\begin{array}{c}\text { Remaining Ener- } \\
\text { gy(Joule) }\end{array}$ & 90.2768 & 126.398 & LOW & HIGH \\
\hline
\end{tabular}




\begin{tabular}{|c|c|c|c|c|}
\hline Throughput[kbps] & 683.96 & 50.63 & HIGH & LOWER \\
\hline $\begin{array}{c}\text { Packet Delivery Ratio } \\
(\%)\end{array}$ & 53.5686 & 86.5303 & LOW & HIGH \\
\hline $\begin{array}{c}\text { End to End Delay } \\
(\text { Sec })\end{array}$ & 141.3474 & 24.6854 & HIGH & LOWER \\
\hline
\end{tabular}

\section{Conclusion}

The work focus was basically comparative evaluation of energy efficiency behaviours of 802.11 and S-MAC by simulating a fixed predecided number of nodes. In this paper, we compared the performance of IEEE 802.11 MAC protocol with S-MAC protocol on energy consumption in network. S-MAC obtains significant energy savings compared with $802.11 \mathrm{MAC}$, which is clearly discussed in the experimented result section. The simulation conclusion shows that the S-MAC with periodic sleep obtains more energy savings compared with IEEE-802.11 protocol. It is able to greatly prolong the network lifetime, which is critical for real world sensor network applications. Periodic sleeping provides excellent energy performance at light traffic load. It makes SMAC with periodic sleep and adaptive listening ideal for sensor networks where traffic is intermittent. In this section you should present the conclusion of the paper. Conclusions must focus on the novelty and exceptional results you acquired. Allow a sufficient space in the article for conclusions. Do not repeat the contents of Introduction or the Abstract. Focus on the essential things of your article.

\section{References}

[1] J. Yick, B. Mukherjeeand D. Ghosal, "Wireless sensor network survey", Computer Networks, Volume 52, Issue 12, August 2008, pp. $2292-2330$

[2] Pei Huang, Li Xiano, Soltani S, M.W Mutka, Ning Xi, "The Evolution of MAC Protocols in Wireless Sensor Networks : A Survey”, IEEE Communication Survey and Tutorials, Volume:15, Issue: 1, pp. 101-120, 2013.

[3] B. Bates, A. Keating, and R. Kinicki, "Energy analysis of four wireless sensor network MAC protocols," in Proceedings of the 6th International Symposium on Wireless and Pervasive Computing (ISWPC '11), pp. 1-6, IEEE, Hong Kong, February 2011.

[4] J. Kabara and M. Calle, "MAC Protocols Used by Wireless Sensor Networks and a General Method of Performance Evaluation", International Journal of Distributed Sensor Networks, Volume 2012 (2012), Article ID 834784, 11 page.

[5] Wei Ye, John Heidemann, and Deborah Estrin, "An energyefficient mac protocol for wireless sensor networks", In Proceedings of the IEEE Infocom, pages 1567-1576, New York, NY, June 2002.

[6] Yadav, Rajesh. "A Survey of MAC Protocols for Wireless Sensor Networks," UbiCC Journal, Volume 4, Number 3, August 2009.

[7] S. Wen-miao, L Yan-ming, and Z. Shu-e, "Research on SMAC protocol for WSN", Wireless Communications, Networking and Mobile Computing, WiCOM '08, 4th International Conference, Oct 2013, pp.1- 4.

[8] Dave, Ankur and Shweta Singh. "Comparative analysis between IEEE 802.11 and S-MAC." 2015 International Conference on Pervasive Computing (ICPC) (2015), pg.1-5

[9] Poonam Kumari et al, "Performance of Energy Efficient S-MAC Protocol for Wireless Sensor Networks" IJCSMC, ISSN 2320088X, Vol. 4, Issue. 7, July 2015, pg.544 - 549

[10] Rendy Munadi, Andini Eksi Sulistyorini, Feblia Ulfah Fauzi S, Tjahjo Adiprabowo, "Simulation and analysis of energy consumption for S-MAC and T-MAC protocols on wireless sensor network" 2015 IEEE Asia Pacific Conference on Wireless and Mobile (APWiMob)Pages: 142 - 146

[11] Smac-users -- Discussions by users of S-MAC web page. http://mailman.isi.edu/mailman/listinfo/smac-users

[12] Energy Model Update in ns-2 web page. http://www.isi.edu/ilense/software/smac/ns2_energy.html

[13] Awk tutorial, http://www.vectorsite.net/tsawk.html. 